\title{
5 Schlussbetrachtung
}

Nachdem nun die Untersuchungen zu den drei Kirchenschriftstellern abgeschlossen sind, gilt es, die Ergebnisse der analytischen Kapitel im Hinblick auf die eingangs gestellten Fragen zusammenzufassen.

\section{Eine einheitliche Position oder eine Vielfalt der Positionen?}

Es hat sich im Verlauf der Untersuchung gezeigt, dass es zumindest nach dem Befund bei den drei behandelten Kirchenschriftstellern tatsächlich verfehlt ist, von einer einheitlichen Haltung der vorkonstantinischen Kirche zur Soldatenfrage zu sprechen. Sowohl die Autoren selbst als auch die vor allem bei Tertullian reflektierten Ansichten anderer Christen spiegeln ein breites Spektrum an Meinungen wider, dass nicht ohne Weiteres auf einen Nenner gebracht werden kann. ${ }^{1}$ Hier ist dem sich möglicherweise anbahnenden ,New Consensus ${ }^{62}$ sicherlich Recht zu geben. Selbst zwei Autoren, die sich in ihren Ergebnissen teilweise sehr nahe kommen, wie Tertullian und Origenes, erreichen diese auf so unterschiedlichen Wegen und von so verschiedenen Grundlagen ausgehend, dass man auch hier nicht einfach von einer gemeinsamen Position sprechen kann.

Es scheint auf der einen Seite in den Kirchen Kreise gegeben zu haben, die dem Heeresdienst von Christen skeptisch bis ablehnend gegenüberstanden. Dabei ist allerdings noch einmal zu unterscheiden, ob ein getaufter Christ in das Heer eintreten wollte oder ob ein Soldat sich zum Christentum hinwandte und getauft werden wollte.

Tertullian war ein Vertreter dieser strengen Sichtweise: Für ihn war es schlechterdings ein Unding, wollte ein getaufter Christ freiwillig in das römische Heer eintreten. Anders sah es aber aus, wenn ein Soldat Christ werden wollte. Diesem stand auch in den Augen Tertullians die Taufe offen und auch wenn der Karthager es eindeutig vorgezogen hätte, dass der frischgetaufte Christ seinen Dienst im Heer beendete, so war er doch unter bestimmten Umständen bereit, ein Verbleiben im Heer zu gestatten. Ein völliges Verbot jeglichen Heeresdienstes wollte oder konnte also auch Tertullian nicht vertreten. Die Anwesenheit zumindest von solchen Soldatenkonvertiten im Heer nutzte er außerdem bereitwillig für seine apologetischen Ziele.

Auf der anderen Seite gab es aber Christen, die bereit waren, zumindest an einem der zwei Punkte wesentlich weiter zu gehen, wahrscheinlich sogar an beiden. Ter-

\footnotetext{
$1 \mathrm{Zu}$ diesem Punkt kann v. a. auf die neueren Arbeiten von J. Shean (SHEAn, Soldiering) und H. Brennecke (BRENNECKE, An fidelis [2007]) verwiesen werden, die jeweils zu demselben Ergebnis kommen, wenn auch zumindest teilweise auf einem anderen Weg.

2 „Division: at least from the end of the second century it is clear that various Christians had different approaches to warfare. Especially there was a divergence in Christian opinion and practice.“' KREIDER, Military Service, 417; vgl. HunTER, Research, 93; SIDER, Killing, 165; s. dazu oben S. $33 f$.
} 
tullian belegt die Existenz einer solchen Gruppe in der karthagischen Kirche. In ihren Augen war es konvertierten Soldaten selbstverständlich erlaubt, im Heer zu verbleiben, und gegebenenfalls erhielten sie sogar einen Dispens von gewissen Vorgaben der kirchlichen disciplina. Es scheint sogar so zu sein, dass diese Gruppierung zur Zeit der Abfassung von De corona die Haltung der Kirche in Karthago zu diesen Fragen bestimmte. Aus dieser Schrift wird ebenfalls erkennbar, dass eine vergleichbare Haltung auch in der römischen Kirche (einflussreich?) vertreten war. Wie aus der Schrift De idololatria außerdem deutlich wird, gab es in Karthago eine zumindest nicht mehr zu ignorierende, vielleicht sogar substantielle und theologisch auf jeden Fall artikulationsfähige Gruppe ${ }^{3}$, die sich für die Möglichkeit des Eintritts von Christen in die Armee stark machte. Außerdem nutzte sie dem Anschein nach die Anwesenheit von Christen im Heer, um unter Hinweis auf jene für die Möglichkeit, städtische Ämter zu übernehmen, zu argumentieren.

Eine Position, die mit derjenigen der innergemeindlichen Gegner Tertullians in wesentlichen Zügen übereinzustimmen scheint, vertrat Clemens in Alexandria. Auch er gestattete selbstverständlich, dass konvertierte Soldaten ihren Dienst fortsetzten. Außerdem stand er wahrscheinlich auch einem möglichen Eintritt getaufter Christen zumindest nicht völlig ablehnend gegenüber. Selbst die Beteiligung an einem - gerechten! - Krieg scheint für ihn vorstellbar gewesen zu sein. Einen direkten Beleg für die Haltung der alexandrinischen Kirche gibt es im Werk des Clemens nicht, es ist allerdings angesichts seiner Rolle als gemeindlicher oder zumindest gemeindenaher Lehrer plausibel, wenn man annimmt, dass er nicht allzu weit von deren Position entfernt war.

Origenes vertrat eine Einstellung zur Soldatenfrage, die im Ergebnis derjenigen Tertullians nahezukommen scheint, wenn er auch insgesamt eine etwas weniger kritische Haltung als der Karthager vertreten haben dürfte. Allerdings findet sich bei ihm nirgendwo die explizite Differenzierung zwischen getauften Eintrittswilligen und soldatischen Konvertiten. Auch er stand einem Eintritt von Christen in die römische Armee ablehnend gegenüber, da ein solcher Schritt mit dem christlichen Bekenntnis beziehungsweise mit der Rolle der Christen in der Welt nur schwer vereinbar war. Es scheint allerdings, sollte sich die hier im Anschluss an G. Caspary vertretene Interpretation des Katenenfragments zu seinem Matthäuskommentar als richtig erweisen, dass er während ihres Heeresdienstes zum Christentum konvertierten Soldaten das Verbleiben in ihrer Stellung ohne größere Vorbehalte gestattete. Auch übte er an keiner Stelle eine mit der Tertullians auch nur annähernd vergleichbare hochpole-

3 Ob diese Gruppe mit der erstgenannten identisch war, ob sie nur eine Teilmenge daraus darstellte oder ob sich noch einmal andere Stimmen einbrachten, diese Frage kann aus den Texten Tertullians nicht beantwortet werden. Es dürfte allerdings wahrscheinlich sein, dass die Befürworter eines Eintritts von getauften Christen in das Heer auch einem Verbleiben konvertierter Soldaten im Heer positiv gegenüberstanden. Es muss daher zumindest größere Überschneidungen zwischen beiden Gruppen gegeben haben. Ob dagegen jeder Befürworter eines Verbleibens konvertierter Soldaten im Heer auch einen Eintritt getaufter Christen unterstütze, ist weit weniger wahrscheinlich. 
mische Kritik an Christen, die bereits im Heer dienten. Diese etwas offenere Haltung dürfte sich nicht ausschließlich inhaltlich-theologischen Überlegungen verdanken, sondern auch der Tatsache geschuldet sein, dass Origenes fast ein halbes Jahrhundert nach Tertullian schrieb. Christen im Heer dürften zu seiner Zeit weiter verbreitet und in den Gemeinden insgesamt anerkannter gewesen sein als zur Zeit des Karthagers. Sie waren eine Realität, der Origenes Rechnung tragen musste.

\section{Die Begründungszusammenhänge bei den Kirchenschriftstellern}

Die Art und Weise allerdings, wie Origenes für seine Haltung argumentierte, unterscheidet sich substantiell von derjenigen Tertullians. Für ihn waren heilsgeschichtlich-eschatologische Überlegungen der entscheidende Hintergrund, warum Christen nach Möglichkeit nicht im Heer des Kaisers dienen sollten. Seit dem Kommen Christi hatte das neue Gottesvolk der Christen eine ganz konkrete, ihrer endzeitlichen Bestimmung geschuldete Rolle zu spielen. In dieser Rolle als priesterliche Gruppierung traten sie dann durchaus für den Kaiser und seine Heere ein und kämpften für ihn im Gebet, wenn er denn einen gerechten Krieg führte. Mit dem Schwert in der Hand kämpften sie aber nicht für ihn, da sie ja ihre Hände frei von Blut halten mussten, um zu ihrem Gott beten zu können.

Bei Origenes spielte der Gedanke, dass Christen sich vom Töten und von der Gewaltanwendung möglichst fernhalten sollten, also eine zentrale Rolle in seiner Argumentation. Nur dass dieser Gedanke nicht auf einer - im modernen Sinn pazifistischen - Ablehnung jeglicher Gewaltausübung beruhte, sondern eben auf der heilsgeschichtlich begründeten Rollenverteilung zwischen dem heidnischen Kaiser und den Christen. Denn der Kaiser durfte durchaus Gewalt ausüben und Krieg führen und die Christen unterstützten ihn dabei sogar mit ihren Gebeten.

In der argumentativen Zuspitzung dieser Position in Contra Celsum erhielten seine Ausführungen dann sogar noch eine gegen den Götzendienst gerichtete Spitze. Allerdings zielte er hier nicht auf die Heeresreligion im engeren Sinn, sondern auf die von Celsus allgemein erhobene Forderung nach Verehrung der Götter und Dämonen, die sich allerdings im Kaisereid und im Dienst in den Heeren und städtischen Ämtern konkretisierte. Der im Heer allgegenwärtige heidnische Kultus im engeren Sinn spielt jedoch in einem Fragment seiner Korintherauslegung eine Rolle, das zeigt, dass ihm dieser Gedanke im Zusammenhang mit der Armee präsent war und für ihn auch ein deutlich erkennbares Gewicht hatte. Diese Passage stellt argumentativ den engsten Berührungspunkt zu Tertullian dar.

Der zentrale Aspekt der ablehnenden Haltung Tertullians war seine Abscheu vor den im Heer allgegenwärtigen heidnischen Kulten, insbesondere vor der seines Erachtens im Kult der signa beispielhaft verkörperten Heeresreligion im engeren Sinn. Was sich bei Origenes nur an einer fragmentarisch überlieferten Stelle fand, nahm bei ihm sowohl in De idololatria als auch in De corona die entscheidende Position in seiner Erörterung ein. In De corona wurde sie sogar äußerst ausführlich argumentativ 
entfaltet. Es waren die allgegenwärtigen Gefahren des Götzendienstes, denen der Christ in der Armee kaum entkommen konnte, die für Tertullian das entscheidende Hindernis für den Heeresdienst der Christen darstellten. Zwar wurde auch das Problem des Blutvergießens zumindest in De corona explizit angesprochen, es war der Idolatrieproblematik aber eindeutig nach- und untergeordnet.

Bei Clemens wurde weder die Heeresreligion noch das Blutvergießen in den erhaltenen Texten zur Soldatenfrage direkt thematisiert. Über die Gründe dafür kann nur spekuliert werden, aber es mag tatsächlich sein, dass er beides im Zusammenhang mit dem Heeresdienst von Christen nicht als entscheidendes Hindernis empfunden hat.

Hier ist eine Stelle, an welcher der ,New Consensus', genauer sein erster Punkt ${ }^{4}$, zumindest einer erneuten Justierung, wenn nicht einer Korrektur bedarf. Auch wenn die Frage des Blutvergießens bei Tertullian (coron. 11) und Origenes (Cels. 8,73) vorkommt, so ist ihr Gewicht vor allem bei Tertullian doch deutlich geringer als das der in verschiedenen Formen auftretenden Idolatrieproblematik. Auch bei Origenes ist die Gewaltproblematik, obwohl prominter als bei Tertullian, deutlich in den weiteren Zusammenhang idolatrischer Fragen eingebettet.

\section{Einheitliche Positionen der jeweiligen Autoren?}

Insgesamt ist es möglich, bei allen drei untersuchten Autoren in der Zusammenschau eine zumindest in groben Zügen einheitliche und stimmige Position herauszuarbeiten. Von einer Unvereinbarkeit der Texte kann also keine Rede sein. Tertullian, Clemens und Origenes hatten jeweils grundlegende Überzeugungen, die sie dann ihren jeweiligen konkreten rhetorisch-argumentativen Zielsetzungen entsprechend pointieren konnten. Es hat sich gezeigt, dass es zumindest grundsätzlich möglich ist, aus den jeweils bekannten Zuspitzungen auf die dahinter stehenden Überzeugungen zurückzugreifen.

Es wurde aber ebenfalls deutlich, dass sich die in ihrer jeweiligen Zielsetzung und Zuspitzung zum Teil sehr disparaten Texte nicht in allen Einzelheiten problemlos und vollständig zusammenbringen lassen. Es bleiben an den Rändern durchaus Fragen offen. Man denke hier nur an das Problem der Einordnung des Katenenfragments zum Matthäuskommentar des Origenes oder die Frage, wie Tertullian oder Origenes zu einer Beteiligung christlicher Soldaten an einem Abwehrkampf gegen einen Einfall äußerer Feinde stehen würden. Sichere Antworten sind nicht an jeder Stelle möglich,

4 „Idolatry: the early Christians were as repelled by the idolatry of the Roman army as they were by killing, if not more so; and the most vocal of early Christian opponents of military service based their objections as much upon their 'abhorrence of Roman army religion' as their rejection of shedding blood.“ Kreider, Military Service, 417; vgl. HunTer, Research, 93; SidER, Killing, 165; s. dazu oben S. $33 f$. 
mitunter bleibt nur, Wahrscheinlichkeiten abzuwägen, sich mit begründeten Vermutungen zu bescheiden und manche Fragen am Ende offen zu lassen.

\section{Frühchristlicher Pazifismus?}

Es gibt aber auch Fragen, die beantwortet werden können. Zu diesen gehört die Frage nach dem sogenannten frühchristlichen Pazifismus der vorkonstantinischen Zeit. Aus den erarbeiteten Ergebnissen wird hinreichend deutlich, dass von einem solchen frühchristlichen Pazifismus im Sinne der zugrunde gelegten Definition bei den besprochenen Autoren nicht die Rede sein kann. Vielmehr wird dem Problem des heidnischen Kultus, der in der römischen Armee fast allgegenwärtig war, vor allem bei Tertullian ein hohes Gewicht beigemessen. Und auch die heilsgeschichtlich-eschatologische Argumentation des Origenes kann man nicht einfach unter diesem modernen Konzept subsumieren. Bei Clemens ist es ohnehin fehl am Platz, von Pazifismus zu sprechen.

Die untersuchten Autoren mögen aus verschiedenen Gründen dem Soldatendienst von Christen in unterschiedlichem Umfang mehr oder weniger skeptisch gegenüber gestanden haben. Pazifisten waren sie allesamt nicht! Es sei noch einmal an die eingangs aufgestellte Definition von Pazifismus erinnert ${ }^{5}$ : Pazifismus beinhaltet erstens die Ablehnung von Krieg oder Kriegsdienst, ggf. sogar von Heeresdienst im Frieden. Diese Ablehnung geschieht zweitens aus moralischen Gründen (Gewaltbeziehungsweise Tötungsverbot). Im speziellen Fall des frühen Christentums ist diese moralisch begründete Ablehnung außerdem Ausdruck einer christlichen Glaubensverpflichtung. Oder wie der Duden festhält: Es handelt sich um eine „weltanschauliche Strömung, die jeden Krieg als Mittel der Auseinandersetzung ablehnt und den Verzicht auf Rüstung und militärische Ausbildung fordert“6. Keiner der untersuchten Kirchenschriftsteller lehnt Krieg als „Mittel der Auseinandersetzung“ ab, lediglich die Beteiligung der Christen daran stand zur Diskussion. Auch forderte keiner von ihnen einen Verzicht auf Rüstung und militärische Ausbildung, im Gegenteil, selbst Tertullian und Origenes beteten für den Sieg der kaiserlichen Heere. Origenes wertet diese Gebete sogar als einen entscheidenden Beitrag zur Kriegführung des Kaisers. Selbst bei der Begründung einer kritischen Haltung zum Heeres- beziehungsweise Kriegsdienst von Christen spielt die Ablehnung von Gewaltanwendung wenn überhaupt nur eine deutlich untergeordnete Rolle. Hier zeigt sich exemplarisch, wie problematisch es sein kann, antike Standpunkte mit modernen Konzepten zu fassen.

5 Siehe oben S. 10

6 Duden GWb, s.v. Pazifismus. 


\section{Kirchenschriftsteller und Kirche(n)?}

Man kann außerdem die untersuchten Autoren nicht ohne weiteres einfach als repräsentativ für die Haltung ihrer jeweiligen Kirchen oder gar die Haltung der Kirche(n) im Ganzen betrachten. Auch hier gibt es Unterschiede hinsichtlich der Nähe der einzelnen Positionen zu den jeweiligen Kirchen vor Ort.

Tertullian befand sich beispielsweise in einer sehr erregten Auseinandersetzung mit wahrscheinlich substantiellen Teilen seiner karthagischen Gemeinde über diese Frage und dürfte insgesamt eine wesentlich kritischere Haltung vertreten haben als diese. Dennoch scheint er auch mit seiner inhaltlichen Verortung noch immer einen Platz in seiner Gemeinde gefunden und mit ihr um die richtige Positionierung gerungen $\mathrm{zu}$ haben.

Clemens und vermutlich auch Origenes standen ihren jeweiligen Gemeinden wahrscheinlich wesentlich näher. Zumindest Clemens war aber auch wesentlich weniger kritisch als Tertullian. Für Origenes dürfte dagegen anzunehmen sein, dass seine Haltung etwas enger war als die der christlichen Gemeinden Alexandrias und Cäsareas.

\section{Die Kirchenschriftsteller als Zeugen für christliche Soldaten}

Vor allem Tertullian hat sich als ein wichtiger Zeuge für die Existenz christlicher Soldaten im späten 2. und beginnenden 3. Jhd. n. Chr. erwiesen. Er überliefert die Geschichte vom Regenwunder im Quadenland, die, wenn auch sicherlich in einseitiger Weise christlich vereinnahmt, auf die Anwesenheit einer zumindest wahrnehmbaren Anzahl Christen in der legio XII aus dem ostanatolischen Melitene in den 170er Jahren hinweist. Außerdem wird in der Art der Aufnahme dieser Geschichte deutlich, dass eine solche Präsenz von Christen in einer römischen Legion kirchlicherseits keiner weiteren Rechtfertigung bedurfte, also wahrscheinlich kein unerhörtes Novum darstellte. Weiterhin belegt Tertullian im Apologeticum die Anwesenheit von zumindest nicht wenigen Christen im afrikanischen Heer im späten 2. Jhd. n. Chr. und in De corona von Christen in der Prätorianergarde im frühen 3. Jhd. n. Chr. Zugleich wird vor allem aus De corona deutlich, mit welchen Schwierigkeiten - vor allem in kultischer Hinsicht - Christen im Heer unter Umständen zu kämpfen hatten, wollten sie ihrem Bekenntnis entsprechend leben, aber auch mit welchem Selbstbewusstsein zumindest einzelne eine Verbesserung ihrer Stellung einforderten.

Clemens ist ebenfalls ein wichtiger Zeuge für die Existenz christlicher Soldaten in den in Ägypten stationierten römischen Einheiten im späten 2. Jhd. n. Chr. Leider wird aus seinen Texten nur sehr wenig deutlich, wie das Leben eines Christen in einer dieser Einheiten aussah.

Liest man die entsprechenden Texte des Origenes, so erfährt man leider so gut wie nichts über die Anwesenheit oder das Leben von Christen in der römischen Armee. Lediglich in zwei Fragmenten der Korintherauslegung wird zumindest indirekt deut- 
lich, dass auch für ihn die allgegenwärtige Idolatrie und Unmoral christliche Soldaten vor ganz eigene Herausforderungen stellten.

Als Zeugen für die Anwesenheit von Christen im römischen Heer zeigen alle drei Kirchenschriftsteller auf ihre je eigene Weise, dass das frühe Christentum spätestens in der Zeit um 200 n. Chr. - jetzt auch von außen erkennbar - in der Gesellschaft des Imperium Romanum angekommen war. Eine Konsequenz dieses Angekommenseins war, dass diese Christen ihren teilweise neuen Platz in dieser Gesellschaft grundlegend durchdenken, Freiräume definieren und Grenzen abstecken mussten. ${ }^{7}$ Gerade Tertullians Schrift De idololatria, die so viele verschiedene Lebensbereiche anspricht, in denen Christen in täglichem Kontakt mit der heidnischen Mehrheitsgesellschaft lebten, legt davon beredtes Zeugnis ab. Die Haltung zum Dienst an Kaiser und Reich im römischen Heer war nur ein Bereich unter zahlreichen anderen, in dem dieses Nachdenken zum Ausdruck kam. Durch die hohe Bedeutung, die dem Heer in der römischen Welt zukam, hatte allerdings genau dieser Bereich hohes Gewicht und annähernd repräsentativen Charakter. Eine ursprünglich marginalisierte Gruppe wie die Christen, die zumindest in Teilen ihren Platz im Heer gefunden hatte, hatte ihn auch in der Gesellschaft gefunden. Mitglieder dieser Gruppe, die diesen Platz unter bestimmten Umständen in Frage stellten und kritisch reflektierten, hinterfragten damit auch im größeren Rahmen die gesellschaftliche Rolle der gesamten Gruppe. Alle hier besprochenen Kirchenschriftsteller reflektierten je auf ihre Weise dieses Ankommen beziehungsweise Angekommensein und die damit verbundenen Aushandlungsprozesse.

\section{Ausblick}

Was bleibt am Ende einer solchen Untersuchung? Die hier vorgelegte detaillierte Analyse der Texte von drei Kirchenschriftstellern aus vorkonstantinischer Zeit kann den Boden für eine weitere Erforschung des Fragenkomplexes von ,Christentum und Soldatenstand' in der Alten Kirche bereiten.

Ausgehend von den Ergebnissen dieser Arbeit und gegebenenfalls noch unter Einbeziehung Cyprians und vielleicht der Traditio Apostolica wären erneut die weiteren Zeugnisse für die Existenz und das Leben christlicher Soldaten, die Inschriften und die Märtyrerakten, zu befragen. Vor allem die weiterführende Arbeit an der 2005 entdeckten Gaianus-Inschrift ${ }^{8}$ kann von den Ergebnissen dieser Studie profitieren.

Außerdem müsste noch einmal neu die hier nur angerissene Frage ${ }^{9}$ untersucht werden, wie das Schweigen der literarischen Quellen über christliche Soldaten zwischen dem in der neutestamentlichen Apostelgeschichte erwähnten Zenturio Corne-

7 Vgl. zu diesen Fragen die exzellente Studie von MüHLEnKamp, Heiden; sowie kürzer SchöLlgEN, Teilnahme; sowie GERSTACKER, De idololatria.

8 Siehe oben S. 306.

9 Siehe oben S. $193 \mathrm{f}$. 
lius Mitte des 1. Jhds. n. Chr. und den Soldaten der legio XII in den 170er Jahren n. Chr. $\mathrm{zu}$ interpretieren ist. Die hier vorgetragenen Ergebnisse dürften die bereits knapp umrissene Sichtweise stützen, dass dieses Schweigen keinesfalls bedeutet, dass es vor den 170er Jahren keine christlichen Soldaten gegeben hat. Vielmehr ist davon auszugehen, dass ihre Zahl zusammen mit der Anzahl der Christen überhaupt vielleicht erst um die Wende vom 2. zum 3. Jhd. n. Chr. eine kritische Masse erreicht hatte, die ihre Existenz in das Blickfeld verschiedener Gruppen innerhalb und außerhalb der Kirchen rückte.

Ein dritter Ansatzpunkt, der sich aus den Ergebnissen dieser Arbeit ergeben könnte, wäre eine erneute Hinwendung zu der Frage nach Kontinuität und Diskontinuität zwischen der vorkonstaninischen und der konstantinisch/nachkonstantinischen Zeit mit Blick auf das Verhältnis von Christentum und Soldatenstand. ${ }^{10}$ Es wurde bereits angedeutet, dass der ,New Consensus ${ }^{‘ 11}$ eine grundsätzliche Kontinuität zwischen Kirchenschriftstellern wie Augustinus, die christlichen Heeresdienst gutheißen, mit zumindest einem Teil der vorkonstantinischen Tradition entdeckt. Hier wäre weiter zu fragen, woran diese späteren Denker jeweils präzise anknüpfen, inwieweit sie Positionen nur fortführen oder weiterentwickeln und wo sie eigene Wege gehen. Außerdem wäre zu fragen, ob auch die dem Heeresdienst gegenüber kritische Position eine Weiterführung erfährt und, falls ja, in welchen Gruppen, bei welchen Autoren und in welcher Weise.

Was darüber hinaus bleibt, ist die Erkenntnis, dass das frühe Christentum einen integralen Bestandteil der römischen Antike darstellt, der von Althistorikern nur um den Preis großer inhaltlicher Verluste aus ihrer Arbeit ausgeklammert werden kann. Kirchenschriftsteller wie Tertullian, Clemens und Origenes sind Menschen der Antike, Römer und Griechen, die im Denken ihrer Zeit verwurzelt sind und selbst wichtige Beiträge zur weiteren Entwicklung dieses Denkens geleistet haben. Sie interagieren mit Vorstellungen und Ideen, Institutionen und Personen ihrer Zeit und bieten dabei mitunter auch eine ganz eigene Perspektive auf ihre Welt, die wahrzunehmen unser Verständnis der Antike nur bereichern kann.

10 Für knapp skizzierte Überlegungen in diese Richtung vgl. z. B. SwIFT, Views, 286-293.

11 Siehe oben, S. $33 \mathrm{f}$. 